\title{
Aspectos diagnósticos y terapéuticos en la infección por SARS-CoV-2 (COVID-19) en pacientes pediátricos
}

\author{
Diagnostic and therapeutic aspects in the infection by \\ SARS-CoV-2, (COVID-19) in Pediatrics
}

Raymundo Rodríguez-Herrera, Mauricio Rivera-Díaz, ${ }^{2}$ Alfonso Huante-Anaya ${ }^{3}$

\section{Resumen}

Los coronavirus de alta patogenicidad infectan, predominantemente, las vías aéreas inferiores y causan neumonía mortal, como sucede con la actual pandemia por el virus SARS-CoV-2, denominada enfermedad por COVID-19, contra la que los humanos no tienen inmunidad preexistente. A partir de su brote, diversos estudios han investigado el COVID-19 en adultos, pero los datos de las características epidemiológicas y clínicas en niños son escasos, quienes parecen manifestar formas leves a moderadas, incluso inadvertidas de la enfermedad. Si bien la mortalidad es realmente muy baja, existe riesgo de subdiagnóstico. Por lo anterior se revisó y seleccionó la información más reciente y útil en el medio acerca de los criterios de diagnóstico y tratamiento para contribuir al conocimiento y tendencia de la morbilidad y mortalidad de esta pandemia en la población pediátrica.

PALABRAS CLAVE: Patogenicidad; coronavirus en humanos; vía respiratoria; SARS-CoV-2; COVID-19; subdiagnóstico; pandemia; pacientes pediátricos.

\section{Abstract}

The high pathogenicity human coronavirus predominantly infects the lower respiratory tract causing fatal pneumonia, as it is the case of the current pandemic due to the SARS-CoV-2 virus denominated COVID-19 illness, to which human beings do not have pre-existing immunity. Since the outbreak, an increasing number of studies had investigated COVID-19 on adults, but epidemiologic and clinic characteristics data on offspring are few, who they seem to develop moderate to mild or silent forms of the illness. Even though the mortality is really low, it does exist the risk that pediatric cases could be underdiagnosed. As mentioned, we had revised and selected the most useful and recent information inside our medical branch about the diagnosis and treatment criteria in order to contribute with the knowledge and tendency of the morbidity and mortality of this pandemic on the pediatric population.

KEYWORDS: Pathogenicity; Human coronavirus; Respiratory tract; SARS-CoV-2; COVID-19; Pandemic; Paediatric population.

\author{
Departamento de Medicina Interna, \\ Instituto Nacional de Pediatría, Ciudad \\ de México. \\ 2 Médico Familiar adscrito al servicio \\ de Urgencias de la Secretaria de Salud \\ de la CD MX. \\ ${ }^{3}$ Nefrólogo Pediatra. Instituto Nacional \\ de Pediatría.
}

Recibido: 18 de agosto 2020

Aceptado: 20 de octubre 2020

Correspondencia

Raymundo Rodríguez Herrera

raymundo.rdzh@gmail.com

Este artículo debe citarse como Rodríguez-Herrera R, Rivera-Díaz $M$, Huante-Anaya A. Aspectos diagnósticos y terapéuticos en la infección por SARS-CoV-2 (COVID-19) en pacientes pediátricos. Acta Pediatr Mex 2020; 41(6): 271-85.

\section{ANTECEDENTES}

Los coronavirus se componen de una gran familia de virus comunes en los seres humanos, que pueden causar varias enfermedades en diferentes animales (camellos, vacas, gatos, perros, aves, cerdos, roedores y murciélagos). Los virus de esta familia se clasifican en cuatro géneros: alfa, beta, gamma y delta. Los coronavirus que infectan a los humanos se encuentran en dos de estos géneros: coronavirus alfa y beta. También se dividen en coronavirus de baja y alta patogenicidad. Los de baja patogenicidad infectan la vía respiratoria superior y provocan enfermedad leve, similar al resfriado. Y los de alta patogenicidad (MERS-CoV, SARS-CoV y SARS-CoV-2) infectan, predominantemente, las vías aéreas inferiores y causan neumonía mortal. 
La neumonía severa causada por los coronavirus patógenos en humanos suele asociarse con rápida replicación del virus, proceso inflamatorio celular masivo y elevada respuesta proinflamatoria de citocinas, quimiocinas, o ambas, que generan una lesión pulmonar aguda y síndrome de dificultad respiratoria aguda (SDRA). ${ }^{1}$ El acrónimo COVID-19 es el nombre designado del inglés: coronavirus disease 2019, una enfermedad infecciosa emergente. Es un problema de salud pública en todo el mundo, causado por un patógeno recientemente identificado: el coronavirus SARSCoV-2, nombre designado al agente viral, por sus siglas en inglés Severe Acute Respiratory Syndrome (SARS), CoV por coronavirus y por existir un SARS-CoV se denominó SARS-CoV-2. ${ }^{2}$

Los primeros casos se identificaron en Wuhan, China, en diciembre de 2019, con diseminación mundial rápida. La Organización Mundial de la Salud declaró al COVID-19 pandemia el 11 de marzo de 2020. ${ }^{3,4,5}$ La enfermedad produce síntomas similares a los de la gripe: fiebre, tos seca, disnea, mialgia y fatiga. En casos graves evoluciona a neumonía, síndrome de dificultad respiratoria aguda, sepsis, choque séptico y muerte (3\%). Hasta la fecha no existe tratamiento específico; las medidas principales consisten en aliviar los síntomas y mantener las funciones vitales. ${ }^{5}$

A partir del inicio de la pandemia, la enfermedad por COVID-19 ha sido extensamente estudiada en la población adulta; no obstante, la información en cuanto a morbilidad y mortalidad en la población pediátrica es escasa, al igual que las características clínicas y epidemiológicas. De hecho, los niños parecen manifestar formas asintomáticas, leves o moderadas de la enfermedad. Si bien se han reportado pocos casos de mortalidad, existe riesgo de subdiagnóstico, quizá debido a que los casos con síntomas leves o asintomáticos no reciben atención médica. ${ }^{6,7}$
El 7 de enero de 2020 los científicos chinos aislaron el nuevo coronavirus y realizaron la secuenciación del genoma, que estuvo disponible para la OMS el 12 de enero del mismo año. Estos hallazgos permiten a los laboratorios de diferentes países desarrollar métodos de detección específicos mediante pruebas de PCR. A partir de entonces la OMS denominó a la enfermedad COVID-19 y para el 11 de marzo se habían registrado más de 100 países con la infección, por lo que declararon la pandemia..$^{8,9}$

El primer caso en México se diagnosticó el 28 de febrero de 2020 y para el 6 de agosto del mismo año se habían confirmado 30,640 casos en pacientes menores de 19 años, que representan $3.9 \%$ del total de casos en el país. Asimismo, en este grupo de edad han ocurrido 270 decesos, que suponen $0.33 \%$ del total de defunciones ocurridas en este periodo, ${ }^{10}$ lo que coincide con lo reportado en otros países.

\section{Fisiopatología}

Los mecanismos fisiopatológicos muestran que, al igual que el SARS y MERS-CoV, el SARS-CoV-2 infecta las células epiteliales de las vías respiratorias y las células THP-1 (una línea celular de monocitos), derivadas de monocitos de sangre periférica, macrófagos y células dendríticas, lo que induce una baja producción de factores antivirales (interferones) y altas concentraciones de citocinas proinflamatorias (IL-1 $\beta$, IL-6, factor de necrosis tumoral y quimiocinas, CCL -2, CCL-3 y CCL-5); de este modo, la producción de IFN-I o IFN- $\alpha$ o $\beta$ representa la respuesta de defensa inmunitaria natural clave contra infecciones virales, mientras que el IFN-I es la molécula diana, con función antiviral en las primeras etapas de la infección.

La liberación retrasada de interferones en las primeras etapas de la enfermedad dificulta la 
respuesta antiviral del huésped; así, el rápido aumento de las citocinas y quimiocinas atrae a las células inflamatorias (neutrófilos y monocitos) y ocurre infiltración excesiva de estas últimas y rápida replicación viral. En consecuencia, se induce la apoptosis de las células epiteliales y endoteliales pulmonares, y lesión pulmonar. ${ }^{11,12}$

Después del daño endotelial de los alvéolos aumenta la concentración del factor de Von Willebrand, con subsiguiente activación del factor tisular y liberación de trombina, para posteriormente iniciar la activación de plaquetas y conversión de fibrinógeno en fibrina, formación de trombos y consumo de los factores de coagulación. La activación del complemento a través de las plaquetas es un mediador clave en la inflamación. Además, la interacción entre macrófagos, monocitos, células endoteliales y linfocitos es decisiva en el efecto procoagulante de las infecciones virales. La elevada concentración de productos de degradación del dímero D y fibrina, además del tiempo prolongado de protrombina, representan parámetros asociados con mal pronóstico en pacientes con SARS-CoV- $2 .{ }^{13}$

De acuerdo con un estudio, se confirmó el daño pulmonar en la autopsia de 38 pacientes fallecidos en Italia, cuyo principal hallazgo fue la identificación de plaquetas y fibrina, con formación de trombos en pequeños vasos arteriales. Esta importante observación coincide con el contexto clínico de coagulopatía por consumo (característica importante en estos pacientes) y representa uno de los principales objetivos del tratamiento. $^{14}$

Con base en esta explicación fisiopatológica, el tratamiento de elección en pacientes con enfermedad grave y crítica por COVID-19 consiste en antiinflamatorios esteroides y anticoagulantes.
Según diversos ensayos, la indicación de glucocorticoides aún se discute. De hecho, en los reportes iniciales del brote en China no los recomendaron, debido al riesgo de retrasar la eliminación del virus y favorecer la sobreinfección bacteriana; no obstante, pueden ser efectivos a dosis bajas en pacientes con respuesta inflamatoria severa. ${ }^{15}$ Pese a estos reportes y con base en los conocimientos actuales, algunas guías de diagnóstico y tratamiento de pacientes con COVID-19 recomiendan la prescripción de metilprednisolona y enoxaparina de forma temprana. ${ }^{16}$

En resumen, los pacientes con infección grave o crítica por COVID-19 comparten las siguientes características: ${ }^{17}$

a) Respuesta inflamatoria exagerada (denominada tormenta de citocinas) y parámetros inflamatorios extremadamente altos de proteína $\mathrm{C}$ reactiva y citocinas proinflamatorias (IL-6, TNF $\alpha$, IL-8).

b) Concentraciones bajas de linfocitos, sobre todo células asesinas naturales (NK) en sangre periférica; la mayor parte de las células inmunitarias infiltradas en la lesión pulmonar son monocitos y macrófagos.

c) Un evento semejante a la vasculitis, hipercoagulación y daño a múltiples órganos.

\section{Manifestaciones clínicas}

En diversas publicaciones se informa que el periodo de incubación del COVID-19 se extiende a 14 días, con promedio de 4 a 5 días desde la exposición hasta el inicio de los síntomas. ${ }^{18,19}$ Un estudio informó que $97.5 \%$ de los pacientes con COVID-19 que desarrollan síntomas lo harán dentro de los 11.5 dias de la infección por SARS-CoV-2. ${ }^{20}$ 
Los signos y síntomas del COVID-19 varían al inicio de la enfermedad y mientras ésta avanza aparecen: fiebre (83-99\%), tos (59-82\%), fatiga (44-70\%), anorexia (40-84\%), disnea (31-40\%), esputo (28-33\%) y mialgias (11-35\%). ${ }^{18,21}$

Se han descrito manifestaciones atípicas, incluso los adultos mayores y las personas con comorbilidades médicas pueden resultar, tardíamente, con fiebre y síntomas respiratorios. En un estudio de 1099 pacientes hospitalizados, la fiebre se manifestó al ingreso en $44 \%$ y más tarde en $89 \%{ }^{18}$ El dolor de cabeza, confusión, rinorrea, dolor de garganta, hemoptisis, vómitos y diarrea son menos comunes (menos de 10\%). 18,21 Algunos pacientes con COVID-19 han experimentado síntomas gastrointestinales (diarrea y náuseas) antes de aparecer la fiebre, y signos y síntomas de la vía respiratoria inferior. ${ }^{22}$ La anosmia o ageusia se han informado, anecdóticamente, al inicio de los síntomas respiratorios; ${ }^{23}$ no obstante, se requiere más información para comprender su papel en la identificación del COVID-19. Dong y colaboradores señalan que los signos y síntomas del COVID-19 en niños son similares a los de los adultos, pero generalmente más leves, como lo demuestra una de las series más grandes, que incluyó 2143 pacientes pediátricos (731 casos confirmados y 1412 sospechosos). Del total de casos, 1213 (56.6\%) eran niños. Más de 90\% de todos los pacientes fueron asintomáticos o tuvieron formas leves a moderadas de la enfermedad. Los casos graves o críticos fueron mucho menores que los registrados en pacientes adultos (5.9 vs 18.5\%). ${ }^{24}$ Otro estudio que analizó 1391 niños, correspondientes a casos positivos o sospechosos, confirmó el diagnóstico en 171 casos, cuyas manifestaciones más frecuentes fueron: tos $(48.5 \%)$, dolor faríngeo $(46.2 \%)$ y fiebre $(41.5 \%)$, esta última con duración promedio de tres días (límites de 1-16 días). Otros síntomas menos frecuentes fueron: diarrea, fatiga, rinorrea, vómito y congestión nasal..$^{25}$
Aunque la mayoría de los niños manifiesta formas leves de COVID-19, los virus pueden persistir en las heces durante mucho tiempo, incluso si están ausentes en las secreciones nasofaríngeas. ${ }^{26,27}$

Los niños menores de 1 año son más vulnerables a SARS-CoV-2, pues en este grupo se ha reportado la mayor tasa de casos graves (10.6\%), que representa $50 \%$ de los casos críticos en el estudio de Dong y colaboradores. Se considera cuadro grave cuando coexisten: disnea, cianosis central y saturación de oxígeno menor de 93\%; y cuadro crítico en caso de síndrome de dificultad respiratoria aguda, choque e insuficiencia multiorgánica (por ejemplo: encefalopatía, insuficiencia cardíaca, alteraciones en la coagulación e insuficiencia renal). ${ }^{24}$

El Comité Chino definió a los recién nacidos sospechosos de infección por COVID-19 y a los nacidos de madres con antecedente de la infección entre 14 días antes y 28 después del parto, o recién nacidos directamente expuestos a personas infectadas con COVID-19, independientemente si manifestaron síntomas o no. No existe evidencia directa de transmisión vertical de madre a hijo, pero los recién nacidos pueden infectarse a través del contacto cercano. ${ }^{28}$

El recién nacido infectado puede estar asintomático o tener síntomas leves o graves. El periodo de incubación varía de 3 a 7 días, y el más corto registrado es de 1 día. ${ }^{29}$ Las manifestaciones clínicas neonatales asociadas con COVID-19, sobre todo en prematuros, suelen ser inespecíficas: distermia, taquipnea, quejido, aleteo nasal, taquipnea, apnea, tos, taquicardia, dificultad para la alimentación, letargo, vómito, diarrea y distensión abdominal. ${ }^{30}$

En esta revisión se describen cuatro recién nacidos de madres infectadas por COVID-19, con 
SARS CoV-2. Su edad fluctuó entre 36 horas y 30 días de vida. Todos tuvieron disnea, vómito, tos y fiebre. Los signos vitales permanecieron estables y los síntomas fueron leves. ${ }^{31}$

En otro estudio, la tomografía de tórax de un recién nacido de 36 horas, aunque tenía signos vitales normales, mostró una sombra nodular de alta densidad en el segmento posterior del lóbulo superior del pulmón derecho. Finalmente, se dio de alta del hospital sin complicaciones a los 16 días de vida. ${ }^{32} \mathrm{Al}$ inicio de la pandemia no se habían notificado casos graves de COVID-19 en recién nacidos.

\section{SÍNDROMES CLÍNICOS ASOCIADOS CON COVID-19}

Con la finalidad de tener criterios homogéneos del COVID-19 en pacientes pediátricos, ésta se clasifica en: ${ }^{32,33}$

\section{Infección asintomática (infección inadvertida):} niños con prueba positiva para SARS-CoV-2, sin evidencia de síntomas clínicos ni alteraciones radiológicas.

Enfermedad leve: pacientes sintomáticos que cumplen con la definición de pacientes con COVID-19. Síntomas inespecíficos: fiebre, fatiga, tos (con o sin expectoración), dolor de garganta, anorexia, malestar general, disnea, congestión nasal, cefalea y mialgias. Menos frecuentes: diarrea, náusea y vómito. Sin evidencia de neumonía viral, hipoxia o sepsis. La mayoría se recupera entre 1 y 2 semanas.

\section{Enfermedad moderada-neumonía}

a) Adolescentes: con o sin fiebre. Datos clínicos de neumonía no severa: tos, aumento en la frecuencia respiratoria según la edad.
El diagnóstico es clínico. No existen datos de neumonía severa ni requerimiento de oxígeno.

b) Niños: datos clínicos de neumonía no severa (tos, dificultad para respirar, respiración acelerada, tiro intercostal). Sin datos de neumonía severa. Los estudios de imagen (radiografía de tórax, ultrasonido, tomografía) confirman el diagnóstico y permiten identificar complicaciones.

\section{Enfermedad severa-neumonía severa}

a) Adolescentes: datos clínicos de neumonía: fiebre, tos, disnea, aumento en la frecuencia respiratoria según la edad, además de uno de los siguientes criterios: frecuencia respiratoria mayor de 30 respiraciones por minuto, insuficiencia respiratoria severa, saturación de oxígeno menor de 90\% respirando al aire ambiente.

b) Niños: datos clínicos de neumonía (tos o dificultad para respirar) y alguno de los siguientes datos: cianosis central, saturación de oxígeno menor de 90\%, dificultad respiratoria (quejido, uso de músculos accesorios, taquipnea), apneas intermitentes; datos de alarma: intolerancia a la vía oral, signos de deshidratación, alteración del estado de conciencia (somnolencia, letargo, coma), crisis convulsivas y respiraciones rápidas.

El diagnóstico más común en pacientes con COVID-19 por enfermedad severa es la neumonía grave. Los estudios de imagen (radiografía de tórax, ultrasonido, tomografía) confirman el diagnóstico de neumonía y permiten identificar las complicaciones. 


\section{Enfermedad crítica}

Los pacientes cursan con insuficiencia respiratoria (requerimiento de ventilación mecánica), síndrome de dificultad respiratoria aguda, choque, síndrome de respuesta inflamatoria sistémica e insuficiencia orgánica múltiple.

\section{Síndrome inflamatorio multisistémico asociado con COVID-19 en niños y adolescentes}

Entre marzo y mayo de 2020 se emitieron alertas por varios grupos de estudio: sociedades, asociaciones, colegios de pediatría y cuidados intensivos pediátricos de varios países de Europa y Estados Unidos, ${ }^{34-36}$ debido al incremento de casos de enfermedad de Kawasaki incompleta o atípica, con mayor resistencia a la gammaglobulina intravenosa, tendencia al síndrome de activación por macrófagos, choque hiperinflamatorio y necesidad de ingreso a cuidados intensivos pediátricos.

La manifestación grave en pacientes pediátricos con COVID-19 ha recibido varios nombres: síndrome pediátrico multisistémico inflamatorio, ${ }^{37}$ "Kawashocky", "Coronasacki" o choque hiperinflamatorio, síndrome inflamatorio pediátrico multisistémico asociado, temporalmente, con SARS-CoV-2 (PIMS-TS), y síndrome multisistémico inflamatorio en niños ${ }^{38,39}$ y MIS-C. ${ }^{40}$ El nombre actual, definido por la OMS, es el de síndrome inflamatorio multisistémico (SIM/MIS) en niños y adolescentes con COVID-19. ${ }^{41}$ Esta definición es para niños y adolescentes de 0 a 19 años, que cumplen con los criterios de fiebre por más de 3 días y dos de los siguientes datos:

- Conjuntivitis bilateral no supurativa o signos de inflamación mucocutánea (boca, manos o pies).
- Hipotensión arterial o choque.

- Hallazgos de disfunción miocárdica, pericárdica, valvular o anormalidades coronarias (datos ecocardiográficos o aumento de troponina-NT-pro BNP).

- Evidencia de coagulopatía (por TP, TPT, concentración elevada de dímero-D).

- Manifestaciones gastrointestinales agudas (diarrea, vómitos, o dolor abdominal).

- Elevación de marcadores inflamatorios: velocidad de eritrosedimentación, proteína C-reactiva o procalcitonina.

- Ausencia de otra causa de inflamación: sepsis bacteriana, síndrome de choque tóxico estafilocócico o estreptocócico.

- Evidencia de COVID-19 (RT-PCR, prueba de antígeno o serología positiva) o contacto probable con pacientes con COVID-19.

En Inglaterra, a mediados de abril de 2020, el informe de ocho pacientes reportó: aumento de los casos en 10 días con las denominaciones de choque hiperinflamatorio, enfermedad de Kawasaki atípica, síndrome de choque por enfermedad de Kawasaki y síndrome de choque tóxico. A diferencia de la mayoría de los niños con enfermedad de Kawasaki, todos eran mayores de cinco años, excepto un caso de 4 años. Estos pacientes tuvieron predominio de síntomas gastrointestinales; todos evolucionaron a choque caliente vasopléjico resistente al volumen, y requirieron soporte inotrópico. Entre otros hallazgos ecocardiográficos, un paciente tuvo dilatación coronaria importante y otro hiperrefringencia pericoronaria. Todos recibieron gammamglobulina por vía intravenosa y antimicrobianos, y 7 de 8 egresaron de la unidad de cuidados intensivos pediátricos entre el día 4 y 6 . Un paciente de 14 años falleció por arritmia, choque resistente y accidente cerebrovascular. EI SARS-CoV-2 fue confirmado en 2 pacientes y en 4 hubo contacto con familiares positivos. 
En Italia ${ }^{42}$ documentaron 10 casos con síntomas semejantes a la enfermedad de Kawasaki "Kawasaki-like"); de estos 5 tuvieron la manifestación clínica clásica y solo 1 de 10 era menor de 5 años. Todos tuvieron elevadas concentraciones de marcadores de inflamación aguda, pero los hemocultivos fueron estériles. La RT-PCR para SARS-CoV2 fue positiva en 2 casos, IgG en 8 e IgM en 3. El ecocardiograma fue anormal en 6 pacientes y 2 tuvieron aneurisma coronario mayor de $4 \mathrm{~mm}$. Todos los pacientes recibieron gammamglobulina por vía intravenosa, 8 metilprednisolona como terapia coadyuvante, 2 ácido acetilsalicílico y 2 inotrópicos. Todos tuvieron reacción satisfactoria al tratamiento, fueron dados de alta del hospital y en la actualidad se encuentran en vigilancia clínica y ecocardiográfica.

Belhadjer y su grupo ${ }^{43}$ analizaron, retrospectivamente, 35 niños hospitalizados con disfunción sistólica aguda ventricular izquierda, choque cardiogénico e inflamación multisistémica en 12 hospitales de Francia y 1 de Suiza. La mediana de edad fue de 10 años, ninguno tuvo cardiopatía de base. Se documentó infección por SARS-CoV-2 en 31 pacientes y antecedente de contacto reciente con familiares, con cuadro viral respiratorio agudo en 37\%. El 80\% tuvo síntomas gastrointestinales y 2 de ellos requirieron, incluso, laparotomía exploradora por abdomen agudo. El $83 \%$ fue admitido directamente en la unidad de cuidados intensivos pediátricos, 80\% tuvo choque cardiogénico y requirió fármacos inotrópicos. El ecocardiograma mostró dilatación de las arterias coronarias en 17\%, sin aneurismas en el primer ecocardiograma. No se registraron defunciones.

Whittaker y sus colaboradores, ${ }^{44}$ en una serie de 58 niños hospitalizados con síndrome inflamatorio multisistémico propuesto por la OMS, reporta- ron diversos signos y síntomas: fiebre, síntomas gastrointestinales, erupción cutánea, lesión del miocardio, choque y aneurismas de las arterias coronarias. Con base en estos datos, los autores consideran que existen tres patrones de enfermedad en los niños hospitalizados con síndrome inflamatorio multisistémico: el primero incluye un grupo de niños con fiebre persistente y concentraciones elevadas de marcadores inflamatorios, sin características de enfermedad de Kawasaki, choque o insuficiencia orgánica; el segundo comprende un grupo con criterios de diagnóstico para enfermedad de Kawasaki, y el tercero con datos de choque y evidencia clínica, ecocardiográfica y por estudios de laboratorio de lesión miocárdica.

De acuerdo con las publicaciones, este síndrome comparte algunas características con otros procesos inflamatorios pediátricos: enfermedad de Kawasaki atípica o incompleta, síndrome de choque tóxico (estreptococo y estafilococo), sepsis bacteriana y síndromes de activación de macrófagos. Incluso muestra ciertas diferencias:

1. Comparada con los casos de enfermedad de Kawasaki clásica, que en 90\% aparece en niños menores de 5 años, la mayor parte de las publicaciones indican sujetos escolares o adolescentes.

2. Suele afectar a personas de ascendencia afroamericana, caribeña e hispana, mientras que la enfermedad de Kawasaki afecta solo a pacientes de ascendencia asiática oriental.

3. Los pacientes comparten similitudes con la enfermedad de Kawasaki, pero tienen más manifestaciones gastrointestinales agudas, con cuadros de dolor abdominal intenso, incluso algunos casos requieren laparotomía exploradora. Existe mayor 
riesgo de complicaciones relacionadas con inflamación multisistémica y disfunción sistólica aguda ventricular izquierda, choque cardiogénico y síndrome de activación por macrófagos.

4. La mayoría de los casos no tiene afectación respiratoria significativa, aún con ventilación mecánica como parte de la estabilización cardiovascular.

5. Todos evolucionan a choque caliente, resistente a la resucitación con volumen; y requieren la administración de aminas para alcanzar la respuesta hemodinámica.

6. Algunos niños tienen edema pleural, pericárdico o ascitis, sugerente de un proceso inflamatorio. Al parecer, la insuficiencia cardiaca puede deberse a edema miocárdico y no a daño miocárdico inflamatorio.

7. La manifestación inicial es muy severa, con tormenta de citocinas similar a la descrita en adultos, que puede provocar disfunción multiorgánica (cardiovascular, respiratoria, renal, hepática, gastrointestinal, hematológica y neurológica).

8. Provisionalmente existen tres patrones de enfermedad en niños hospitalizados con síndrome inflamatorio multisistémico: el primer grupo con fiebre persistente y concentraciones elevadas de marcadores inflamatorios, sin características de enfermedad de Kawasaki, choque o insuficiencia orgánica; el segundo grupo con criterios diagnósticos de enfermedad de Kawasaki y el tercero con choque y evidencia clínica, ecocardiográfica y de laboratorio de lesión miocárdica. ${ }^{44}$
9. Los estudios de laboratorio reportan neutrofilia, linfopenia, inflamación más intensa (elevación de proteína $\mathrm{C}$ reactiva, dímero D, fibrinógeno, procalcitonina y ferritina) y concentraciones extremadamente elevadas de marcadores cardiacos que los detectados en pacientes con enfermedad de Kawasaki (troponina, proBNP y NT-proBNP).

10. La ecocardiografía puede mostrar dilatación coronaria, hiperrefringencia pericoronaria o aneurismas coronarios.

11. La prueba de RT-PCR para SARS-CoV-2 puede resultar positiva o negativa, al igual que la detección de anticuerpos IgG, IgM, o ambos.

12. La mayoría de los pacientes tiene reacción satisfactoria a la administración de gammaglobulina, corticosteroides, o ambos. La anakinra, un antagonista del receptor de IL-1, rara vez se indica. Otro posible tratamiento es tozilizumab, dirigido contra el receptor de interleucina-6.

13. El aumento de marcadores de inflamación aguda, incluida la proteína $C$ reactiva, ferritina, troponina, dímero D, proBNP y NT-proBNP pueden ser útiles para predecir la evolución de la enfermedad.

14. En general, se niegan síntomas anteriores de COVID-19 y a menudo muestran un resultado negativo de reacción en cadena de la polimerasa, incluso de anticuerpos positivos o exposición familiar, y la manifestación del síndrome inflamatorio multisistémico después de un retraso de 3 a 6 semanas sugiere que el SARS-CoV-2 
funciona como desencadenante, lo que indica dos tipos de enfermedades.

15. Se desconoce el tratamiento de primera línea, porque se requiere mayor cantidad de casos y ensayos clínicos controlados.

\section{ESTRATEGIAS DE TRATAMIENTO}

En el marco de la evidencia científica disponible y con los resultados de tratamiento incipientes con los antivirales, además de carecer de una vacuna para afrontar la pandemia del COVID-19, la propuesta razonable de la Eastern Medical School para disminuir los efectos de esta infección viral suponen: ${ }^{45}$

- Mejorar la respuesta inmunitaria de los pacientes pediátricos sanos.

- Los pacientes con COVID-19, con síntomas leves a moderados, deben recibir medicamentos de uso cotidiano, con efectividad y seguridad comprobada en otros padecimientos y con alta posibilidad de controlar o disminuir el SARS-CoV-2.

En pacientes adultos y pediátricos con COVID-19 se informan poco más de 20 medicamentos: inmunoglobulina humana, interferones, cloroquina, hidroxicloroquina, arbidol, remdesivir, favipiravir, oseltamivir, talidomida, metilprednisolona y bevacizumab. Los primeros fármacos prescritos fueron la hidroxicloroquina y azitromicina combinados, con excelente reacción clínica contra SARS-CoV-2; sin embargo, se requieren estudios más extensos y con mayor cantidad de pacientes para confirmar estos resultados.

La cloroquina e hidroxicloroquina son medicamentos antipaludismo, con acción antiviral mediante los siguientes mecanismos: interferencia de la glucosilación del receptor ACE2, que utiliza el virus para unirse a las células; inhibición de la enzima quinona reductasa, implicada en la síntesis de ácidos siálicos, que funcionan como ligandos para el virus; alcalinización de los endosomas e inhibición de cinasas (MAPK, entre otras). ${ }^{46,47}$

Además de las características citadas, la hidroxicloroquina tiene efecto inmunomodulador, quizá relacionado con su acción en los receptores tipo toll (TLRs) y disminución de la producción de IL-6 que, fisiopatológicamente, puede ser efectiva en la infección por SARSCoV2, sobre todo en pacientes con síndrome de activación de macrófagos, de extremada gravedad por su frecuente evolución a insuficiencia multiorgánica. ${ }^{48}$ De acuerdo con un estudio, todos los pacientes con COVID-19 que recibieron hidroxicloroquina y azitromicina fueron virológicamente curados versus $57.1 \%$ que solo recibieron hidroxicloroquina, y $12.5 \%$ del grupo control. En la mayoría de los pacientes con COVID-19, la hidroxicloroquina eliminó de la nasofaringe el SARS-CoV-2, entre 3 y 6 días. ${ }^{49}$

Estos resultados son de gran importancia, porque un artículo chino reciente demostró que la duración media de la eliminación viral en pacientes con COVID-19 fue de 20 días (incluso 37 días como periodo más largo). ${ }^{50}$ Los resultados preliminares de Gautret y sus coautores ${ }^{51}$ sugieren un efecto sinérgico de la combinación de hidroxicloroquina y azitromicina. La azitromicina es activa in vitro contra el virus del Zika y Ébola, ${ }^{51} \mathrm{y}$ previene infecciones graves de la vía respiratoria en pacientes infectados. ${ }^{52}$

Los efectos adversos deben vigilarse con electrocardiograma para descartar la prolongación del intervalo QT o bradicardia. Las dosis de hidroxicloroquina, cloroquina y azitromicina recomendadas son: ${ }^{52,53}$ 
1. Hidroxicloroquina: Niños: 3 a $5 \mathrm{mg} / \mathrm{kg} / \mathrm{día}$ por vía oral cada 12-24 horas por 5 a 10 días (máximo 7 mg/kg/día o 400/día). ${ }^{54}$ Adultos: $400 \mathrm{mg} / 12 \mathrm{~h}$ el primer día, por vía oral, seguido de $200 \mathrm{mg} / 12 \mathrm{~h}$ durante 4 días.

2. Cloroquina: $500 \mathrm{mg} / 12 \mathrm{~h}$, por vía oral, durante 7 días.

3. Azitromicina: $500 \mathrm{mg}$ el primer día, seguido de $250 \mathrm{mg} /$ día los cuatro días siguientes.

La gammaglobulina por vía intravenosa es efectiva en diferentes trastornos autoinmunitarios resistentes a los inmunosupresores de referencia. Los anticuerpos ampliamente neutralizantes pueden reconocer diversas glucoproteínas en la superficie de los virus o cubierta proteica de algún virus no envuelto. El ensayo NCT04261426 $6^{55}$ evalúa la función de la gammaglobulina en pacientes con neumonía por COVID-19 y, recientemente, en el síndrome inflamatorio multisistémico asociado con COVID-19 en niños y adolescentes. ${ }^{41-44}$

Tratamiento antiviral específico. No existe ningún antiviral específico efectivo en pacientes con infección por coronavirus, SARS o MERS. Existen diversos fármacos que se indican de manera experimental. ${ }^{56}$

IFN- $\alpha$ por inhalación. Es un antiviral de amplio espectro, prescrito en pacientes con virus de la hepatitis B. Se indica en conjunto con lopinavirritonavir en adultos y se ha iniciado un ensayo clínico para comprobar su eficacia en niños.

Las Guías Chinas recomiendan la nebulización con IFN-2 $\beta$, en dosis de 100,000-200,000 UI/ $\mathrm{kg} / 12 \mathrm{~h}$ para casos leves y de 200,000-400,000 $\mathrm{UI} / \mathrm{kg} / 12 \mathrm{~h}$ para casos severos durante 5 a 7 días. ${ }^{57}$
Lopinavir-ritonavir. Son inhibidores de la proteasa del VIH, incluso ritonavir es un inhibidor del citocromo P450 y GP. Esta combinación se prescribe, frecuentemente, comparada con otros medicamentos para COVID-19; sin embargo, un ensayo efectuado en 199 pacientes con SARSCoV-2, confirmada por laboratorio, informó que lopinavir-ritonavir no demostró mejoría clínica versus los procedimientos de atención establecidos. ${ }^{58,59}$

Remdesivir. Puede considerarse el mejor medicamento para el tratamiento del COVID-19. Es un antiviral de amplio espectro, indicado para el MERS y SARS. ${ }^{60,61}$

Es un fármaco experimental, aún sin autorización ni aprobación médica; sin embargo, puede solicitarse para uso compasivo. El mecanismo antiviral de remdesivir es la interrupción de la cadena retardada del ARN viral naciente. Wang y sus colaboradores demostraron la efectividad del fármaco en pacientes con SARS-CoV-2, en las células Vero E6 (EC90 1.76 $\mu \mathrm{M})$. Un estudio mostró que remdesivir y el IFN- $\beta$ fueron superiores a lopinavir-ritonavir e IFN- $\beta$, tanto in vitro como en un modelo de ratón MERS-CoV. El medicamento ha completado la fase III de ensayo clínico en pacientes con infección del virus Ébola, y los datos de farmacocinética y farmacodinamia están relativamente completos. Sin embargo, la eficacia y seguridad de remdesivir en pacientes con COVID-19 aún deben confirmarse. En Estados Unidos, el tratamiento prescrito solo en un caso demostró resultados satisfactorios. ${ }^{62,63}$

En la actualidad se llevan a cabo dos ensayos clínicos aleatorizados, controlados y doble ciego para evaluar la eficacia y seguridad de remdesivir en 308 y 452 pacientes adultos hospitalizados 
con enfermedad respiratoria por COVID-19 leve a moderada y grave, respectivamente. Remdesivir se administró en dosis de carga de 200 mg el día 1 de tratamiento, seguido de 100 mg/día, por vía intravenosa, durante 9 días en adultos; esta misma dosis se prescribe en pacientes pediátricos mayores de $40 \mathrm{~kg} \cdot{ }^{64,65}$

En pacientes pediátricos menores de $40 \mathrm{~kg}$, la dosis es de $5 \mathrm{mg} / \mathrm{kg}$ el primer día, seguido de una dosis de mantenimiento de $2.5 \mathrm{mg} / \mathrm{kg} / \mathrm{d}$ áa, desde el día 2 al 9, por vía intravenosa.

El efecto clínico de remdesivir en pacientes con COVID-19 aún se desconoce, y los científicos esperan los resultados finales de estos ensayos en curso.

Arbidol. También conocido como umifenovir, está aprobado en Rusia y China para el tratamiento de la gripe y otras infecciones virales; no provoca efectos adversos significativos y está patentado para el tratamiento del SARS. ${ }^{66}$

Oseltamivir. Inhibe la neuraminidasa viral y, en consecuencia, bloquea la liberación de partículas virales de las células huésped, reduciendo la propagación en la vía respiratoria. ${ }^{67}$ Este fármaco se prescribió durante la epidemia del COVID-19 en China, ya sea en combinación o no con antibióticos y corticosteroides. ${ }^{68}$ Oseltamivir se ha evaluado en un ensayo clínico, combinado con cloroquina y favipiravir. ${ }^{69}$ No está clara su eficacia y, posiblemente, se ha indicado para la coinfección con gripe.

Esteroides. La prescripción de esteroides aún se discute y su recomendación se basa en estudios efectuados en pacientes con SARS, en quienes disminuyeron los síntomas y la evolución de la enfermedad, no así los días de estancia hospitalaria. Puede indicarse metilprednisolona en pacientes con evolución rápida de la enfermedad o manifestaciones graves: síndrome de activación por macrófagos durante periodos cortos (3-5 días). La dosis máxima recomendada es de 1-2 mg/kg/ día. ${ }^{70}$ Recientemente se ha indicado en pacientes con síndrome inflamatorio multisistémico asociado con COVID-19 en niños y adolescentes..$^{41-44}$

Tocilizumab. Es un anticuerpo monoclonal dirigido contra el receptor de IL-6, también prescrito para detener la tormenta de citocinas desencadenada en los casos graves de COVID-19. Este fármaco permanece en investigación y se ha planteado como posible tratamiento en pacientes pediátricos graves, internados en la unidad de cuidados intensivos; no existen datos en pacientes menores de 2 años y se sugiere llevar a cabo la determinación de IL-6 luego de 24 horas de la última dosis administrada. Las dosis recomendadas son: en menores de $30 \mathrm{~kg}: 12 \mathrm{mg} /$ $\mathrm{kg}$ por vía intravenosa (diluir en $50 \mathrm{~mL}$ de solución fisiológica al $0.9 \%$ y administrar en 1 hora) y mayores de $30 \mathrm{~kg}: 8 \mathrm{mg} / \mathrm{kg}$ por vía intravenosa (diluir en $100 \mathrm{~mL}$ de solución fisiológica al 0.9\% y administrar en 1 hora); la dosis máxima es de 800 mg por infusión..$^{70-73}$

\section{OTROS TRATAMIENTOS}

Vitamina C. Tiene actividad antioxidante y puede reducir el estrés oxidativo y la inflamación, efectos que aumentan la síntesis de los vasopresores, mejoran la función de las células inmunitarias y la función endovascular, y generan modificaciones inmunológicas epigenéticas. Los ensayos clínicos han reportado datos prometedores en cuanto a reducción de la mortalidad por sepsis; sin embargo, se requieren estudios más extensos para validar estas conclusiones. ${ }^{74}$

Bromhexina. Es un inhibidor de la serina proteasa transmembranal, dicha proteasa es 
responsable de la activación de la glucoproteína $S$ del SARS-CoV y MERS-CoV para que el virus penetre a las células del huésped a través de la membrana plasmática. Actualmente se evalúa su eficacia en combinación con el tratamiento de referencia de pacientes con COVID-19. ${ }^{75}$

\section{Plasma de pacientes curados de COVID-19.} Contiene anticuerpos que ayudan a combatir la enfermedad. El tratamiento experimental está aprobado por la FDA para administrarse en casos de urgencia o riesgo de muerte por COVID-19. ${ }^{76,77}$

\section{CONCLUSIONES}

Con base en lo descrito, la esperanza para el control de la pandemia es lograr la vacuna contra el SARS-CoV-2. Al respecto, el Instituto Nacional para la Investigación en Salud del Reino Unido, además de la participación de la Universidad de Oxford y otras instituciones científicas, ha desarrollado con éxito una vacuna que se encuentra en fase 3 de experimentación en varios países. ${ }^{78}$ Estados Unidos, en coordinación con otros países, lleva a cabo esfuerzos para este cometido. En el marco de la pandemia, y debido a la falta de tratamientos efectivos, además de una vacuna que evite el contagio y la trasmisión del COVID-19, es razonable que todos los médicos hagan uso de manera responsable e informada de los recursos que tengan a su alcance y permanezcan atentos a las nuevas alternativas de prevención y tratamiento en beneficio de los pacientes.

\section{REFERENCIAS}

1. Channappanavar R, et al. Pathogenic human coronavirus infections: causes and consequences of cytokine storm and immunopathology. Semin Immunopathol 2017; 39: 529539. https://doi.org/10.1007/s00281-017-0629-x

2. World Health Organization. Naming the Coronavirus Disease (COVID-19) and the Virus That Causes It. 2020. www. who.int/emergencies/diseases/novel-coronavirus-2019/
3. Epidemiological characteristics of an outbreak of 2019 novel coronavirus diseases (COVID-19) in China. Zhonghua Liu Xing Bing Xue Za Zhi 2020; 41 (2): 145-151. doi. 10.3760/cma.j.issn.0254-6450.2020.02.003

4. Aviso Epidemiológico Enfermedad COVID-19 por SARSCoV-2 (17.03.20) Disponible en: https://www.gob.mx/ cms/uploads/attachment/file/541794/AE_Enfermedad_COVID-19_SARS-CoV-2_2020.03.17.pdf

5. Coronavirus (COVID-19)-Comunicado Técnico Diario. (Consulta: marzo de 2020). Disponible en: https://www. gob.mx/cms/uploads/attachment/file/543968/Comunicado_Tecnico_Diario_COVID-19_2020.03.27.pdf

6. van Doremalen $\mathrm{N}$, et al. Aerosol and surface stability of SARS-CoV-2 as compared with SARS-CoV-1. N Engl J Med 2020; 382 (16): 1564-1567. doi. 10.1056/NEJMc2004973

7. Li R, et al. Substantial undocumented infection facilitates the rapid dissemination of novel coronavirus (SARSCoV-2). Science 2020; 368 (6490): 489-493. doi. 10.1126/ science.abb3221

8. World Health Organization. Naming the Coronavirus Disease (COVID-19) and the Virus That Causes It. 2020, www.who.int/emergencies/diseases/novel-coronavirus-2019/

9. Noticias ONU. El coronavirus es una pandemia. Disponible en: https://news.un.org/es/story/2020/03/1470991

10. Covid-19 México. Disponible en: https://coronavirus. gob. $\mathrm{mx} /$ datos/

11. Zhang Y, et al. Treat 2019 novel coronavirus (COVID-19) with IL-6 inhibitor: Are we already that far? Drug Discov Ther 2020; 14 (2): 100-102. doi. 10.5582/ddt.2020.03006. PMID: 32378647.

12. Qing $Y$, et al. The pathogenesis and treatment of the "Cytokine Storm" in COVID-19. J Infect 2020; 80 (6): 607-613. doi. https://doi.org/10.1016/j.jinf.2020.03.037

13. Giannis D, et al. Coagulation disorders in coronavirus infected patients: COVID-19, SARS-CoV-1, MERS-CoV and lessons from the past. J Clin Virol 2020; 127: 104362. doi. 10.1016/j.jcv.2020.104362

14. Carsana L, et al. Pulmonary post-mortem findings in a large series of COVID-19 cases from northern Italy. Med Rxiv 2020. doi. https://doi. org/10.1101/2020.04.19.20054262

15. Li T, et al. Clinical observation and management of COVID-19 patients. Emerg Microbes Infect 2020; 9 (1): 687-690. doi. 10.1080/22221751.2020.174132

16. Li T. Diagnosis and clinical management of severe acute respiratory syndrome Coronavirus 2 (SARS-CoV-2) infection: an operational recommendation of Peking Union Medical College Hospital (V2.0). Emerg Microbes Infect 2020; 9 (1): 582-585. doi.10.1080/22221751.202 0.1735265

17. Zhang $\mathbf{W}$, et al. The use of anti-inflammatory drugs in the treatment of people with severe coronavirus disease 2019 (COVID-19): The Perspectives of clinical immunologists from China. Clin Immunol 2020; 214: 108393. doi. 10.1016/j.clim.2020.108393 
Rodríguez-Herrera R, et al. SARS-CoV-2 (COVID-19) en pacientes pediátricos

18. Fu L, et al. Clinical characteristics of coronavirus disease 2019 (COVID-19) in China: A systematic review and metaanalysis. J Infect 2020; 80 (6): 656-665. doi. 10.1016/j. jinf.2020.03.041

19. Li Q, et al. Early transmission dynamics in wuhan, china, of novel coronavirus-infected pneumonia. N Engl J Med 2020; 382 (13): 1199-1207. doi. 10.1056/NEJMoa2001316

20. Lauer $S$, et al. The incubation period of Coronavirus disease 2019 (COVID-19) From publicly reported confirmed cases: Estimation and application. Ann Intern Med 2020; 172 (9): 577-582. doi. 10.7326/M20-0504

21. National Center for Immunization and Respiratory Diseases (NCIRD), Division of Viral Diseases. Interim Clinical Guidance for Management of Patients with Confirmed Coronavirus Disease (COVID-19). 2020. https://www.winfocus.org/ interim-clinical-guidance-for-management-of-patientswith-confirmed-coronavirus-disease-covid-19/

22. Pan L, et al. Clinical characteristics of COVID-19 patients with digestive symptoms in Hubei, China: a descriptive, cross-sectional, multicenter study. Am J Gastroenterol 2020; 115 (5): 766-773. https://www.unboundmedicine.com/medline/citation/32287140/Clinical_Characteristics_of_COVID_19_Patients_With_Digestive_Symptoms_in_Hubei_China:_A_Descriptive_Cross_Sectional_Multicenter_Study_

23. Giacomelli A, et al. Self-reported Olfactory and Taste Disorders in Patients With Severe Acute Respiratory Coronavirus 2 Infection: A Cross-sectional Study. Clin Infect Dis 2020; 71 (15): 889-890. doi. 10.1093/cid/ciaa330

24. Dong $\mathrm{Y}$, et al. Epidemiological characteristics of 2143 pediatric patients with 2019 coronavirus disease in China. Pediatr 2020; 58 (4): 712-713. doi. 10.1542/peds.2020-0702

25. Lu X, et al. SARS-CoV-2 Infection in Children. N Engl J Med 2020; 382 (17): 1663-1665. doi. 10.1056/NEJMc2005073.

26. Tang A, et al. Detection of Novel Coronavirus by RT-PCR in Stool Specimen from Asymptomatic Child, China. Emerg Infect Dis 2020; 26 (6): 1337-1339. doi. 10.3201/ eid2606.200301.

27. Jiehao C, et al. A case series of children with 2019 novel coronavirus infection: Clinical and epidemiological features. Clin Infect Dis 2020; 71 (6): 1547-1551. doi. 10.1093/ $\mathrm{cid} / \mathrm{ciaa} 198$.

28. Tinku J, et al. International Pulmonologist's. Consensus On Covid-19. https://amcichac.org/wp-content/ uploads/2020/04/PulmonolConsens2Ed.Covid_.pdf

29. Chen $Z$, et al. Diagnosis and treatment recommendations for pediatric respiratory infection caused by the 2019 novel coronavirus. World J Pediatr 2020; 16 (3): 240-246. doi. 10.1007/s12519-020-00345-5.

30. Wang L, et al. Working Committee on Perinatal and Neonatal Management for the Prevention and Control of the 2019 Novel Coronavirus Infection. Chinese expert consensus on the perinatal and neonatal management for the prevention and control of the 2019 novel coronavirus infection (First edition). Ann Transl Med 2020; 8 (3): 47. doi. 10.21037/ atm.2020.02.20.
31. Paraluppi V, et al. COVID-19 in newborns and in children: the state of the art. J Pediatr Neonat Individual Med 2020; 9 (1): e090138. doi. 10.7363/090138.

32. Shen K, et al. Diagnosis, treatment, and prevention of 2019 novel coronavirus infection in children: experts' consensus statement. World J Pediatr 2020; 16 (3): 223231. doi. 10.1007/s12519-020-00343-7.

33. World Health Organization. (2020). Clinical management of COVID-19: interim guidance. 2020. https://apps.who. int/iris/handle/10665/332196.

34. Royal College of Paediatrics and Child Health. Guidance: paediatric multisystem inflammatory syndrome temporally associated with COVID-19. (Consulta: mayo 2020). https://www.rcpch.ac.uk/resources/guidance-paediatric/ multisystem inflammatory-syndrome-temporally-associatedcovid-19

35. Asociación Española de Pediatría. Información para las familias respecto al comunicado interno "Alerta sobre casos de shock pediátrico" difundido en redes sociales. https://www.aeped.es/noticias/informacion-las-familiasrespecto-al-comunicado-interno-alerta-sobrecasosshock-pediatrico

36. NYC Health. 2020 Health Alert \#13: Pediatric Multi-System Inflammatory Syndrome Potentially Associated with COVID-19. https://www1.nyc.gov/assets/doh/downloads/ pdf/han/alert/2020/covid-19-pediatric-multi-systeminflammatory-syndrome.pdf

37. Ulloa GR, et al. Síndrome inflamatorio multisistémico asociado a COVID-19 en niños y adolescentes: un llamado al diagnóstico. Rev Chilena Infectol. 2020; 37 (3): 199-201. https://scielo.conicyt.cl/scielo.php?script=sci_arttext\&p id=S0716-10182020000300199

38. Hennon T, et al. COVID-19 associated Multisystem Inflammatory Syndrome in Children (MIS-C) guidelines; a Western New York approach. Prog Pediatr Cardiol. 2020: 101232. doi. 10.1016/j.ppedcard.2020.101232.

39. Riphagen $\mathrm{S}$, et al. Hyperinflammatory shock in children during COVID-19 pandemic. Lancet 2020; 395 (10237): 1607-1608. doi. 10.1016/S0140-6736(20)31094-1.

40. Centers for Disease Control and Prevention. Emergency preparedness and response: health alert network. https://emergency.cdc.gov/han/ 2020/han00432.asp

41. World Health Organization. Multisystem Inflammatory Sindrome in Children and adolescents temporally related to COVID-19. https://www.who.int/news-room/commentaries/detail/multisystem-inflammatory-syndrome-inchildren and-adolescents-with-covid-19

42. Verdoni L, et al. An outbreak of severe Kawasaki-like disease at the Italian epicentre of the SARS-CoV-2 epidemic: an observational cohort study. Lancet 2020; 395 (10239): 1771-1778. doi. 10.1016/S0140-6736(20)31103-X.

43. Belhadjer Z, et al. Acute heart failure in Multisystem Inflammatory Syndrome in Children (MIS-C) in the context of global SARS-CoV-2 pandemic. Circulation 2020. doi. 10.1161/CIRCULATIONAHA.120.048360. 
44. Whittaker E, et al. PIMS-TS Study Group and EUCLIDS and PERFORM Consortia. Clinical Characteristics of 58 Children with a Pediatric Inflammatory Multisystem Syndrome Temporally Associated With SARS-CoV-2. JAMA 2020; 324 (3): 259-269. doi. 10.1001/jama.2020.10369.

45. Marik P. EVMS. Critical care Covid-19 management protocol. https://www.evms.edu/covid-19/medical_information_resources/

46. Devaux CA, et al. New insights on the antiviral effects of chloroquine against coronavirus: what to expect for COVID-19? Int J Antimicrob Agents 2020: 105938. doi. 10.1016/j.ijantimicag.2020.105938

47. Sahraei Z, et al. Aminoquinolines against Coronavirus Disease 2019 (COVID-19): chloroquine or hydroxychloroquine. Int J Antimicrob Agents 2020; 55 (4): 105945. doi. 10.1016/j.ijantimicag.2020.105945

48. Lasheras I, et al. Uso de antipalúdicos en el tratamiento del COVID-19: ¿una ventana de oportunidad? Med Clín 2020: 155 (1):23-25. doi. https://doi.org/10.1016/j.medcli.2020.04.004

49. Gautret $P$, et al. Hydroxychloroquine and azithromycin as a treatment of COVID-19: results of an open-label non-randomized clinical trial. Int J Antimicrob Agents 2020; 56 (1): 105949. doi. 10.1016/j.ijantimicag.2020.105949

50. Zhou F, et al. Clinical course and risk factors for mortality of adult inpatients with COVID-19 in Wuhan, China: a retrospective cohort study. Lancet 2020; 395 (10229): 1054-1062. doi. 10.1016/S0140-6736(20)30566-3.

51. Bosseboeuf $E$, et al. Azithromycin inhibits the replication of Zika virus. J Antivirals Antiretrovirals 2018; 10 (1): 6-11. doi. 10.4172/1948-5964.1000173.

52. Bacharier LB, et al. Early administration of azithromycin and prevention of severe lower respiratory tract illnesses in preschool children with a history of such illnesses: A randomized clinical trial. JAMA 2015; 314 (19): 2034-2044. doi. 10.1001/jama.2015.13896

53. Hospital Universitario de Getafe. Manejo de pacientes con COVID-19. Versión 3: 24 Marzo 2020. https://arydol. com/wp-content/uploads/2020/04/PROTOCOLO-DEURGENCIAS-2.pdf

54. Sankar J, et al. COVID-19 in Children: Clinical Approach and Management. Indian J Pediatr 2020; 87 (6): 433-442. doi. 10.1007/s12098-020-03292-1

55. Peking Union Medical College Hospital. The Efficacy of Intravenous Immunoglobulin Therapy for Severe 2019nCoV Infected Pneumonia. https://clinicaltrials.gov/ct2/ show/NCT04261426

56. Lu H. Drug treatment options for the 2019-new coronavirus (2019-nCoV). Biosci Trends 2020; 14 (1):69-71. doi. 10.5582/bst.2020.01020.

57. Schneider WM, et al. Interferon-Stimulated Genes: A Complex Web of Host Defenses. Annu Rev Immunol 2015; 32: 513-45. doi. 10.1146/annurev-immunol-032713-120231.

58. Bin C, et al. A Trial of lopinavir-ritonavir in adults hospitalized with severe Covid-19. N Eng J Med 2020; 382 (19): 1787-1799. doi. 10.1056/NEJMoa2001282
59. World Health Organization. "Solidarity" clinical trial for COVID-19 treatments. https://www.who.int/emergencies/ diseases/novel-coronavirus-2019

60. Mulangu S, et al. A Randomized, Controlled Trial of Ebola Virus Disease Therapeutics. N Engl J Med 2019; 381 (24): 2293-2303. doi. 10.1056/NEJMoa1910993

61. Cao $\mathrm{Y}$, et al. Remdesivir for severe acute respiratory syndrome coronavirus 2 causing COVID-19: An evaluation of the evidence. Travel Med Infect Dis 2020; 35: 101647. doi. 10.1016/j.tmaid.2020.101647.

62. Agostini $\mathrm{M}$, et al. Coronavirus susceptibility to the antiviral remdesivir (GS-5734) is mediated by the viral polymerase and the proo freading exoribonuclease. mBio 2018; 9 (2): e00221-18. https://mbio.asm.org/content/9/2/e00221-18

63. Holshue $\mathbf{M}$, et al. First case of 2019 novel coronavirus in the United States. N Engl J Med 2020; 382 (10): 929-936. doi. 10.1056/NEJMoa2001191.

64. Al-Tawfiq J, et al. Remdesivir as a possible therapeutic option for the COVID-19. Travel Med Infect Dis 2020; 34: 101615. doi. 10.1016/j.tmaid.2020.101615.

65. Wang $\mathrm{M}$, et al. Remdesivir and chloroquine effectively inhibit the recently emerged novel coronavirus (2019nCoV) in vitro. Cell Res 2020; 30 (3): 269-271. doi. 10.1038/ s41422-020-0282-0.

66. Jeiming QJ. Clinical Study of Arbidol Hydrochloride Tablets in the Treatment of Pneumonia Caused by Novel Coronavirus. 2020. https://clinicaltrials.gov/ct2/show/ NCT04260594

67. Uyeki T. Oseltamivir Treatment of Influenza in Children. Clin Infect Dis 2018; 66 (10): 1501-3. doi. 10.1093/cid/cix1150

68. Wang D, et al. Clinical characteristics of 138 hospitalized patients with 2019 novel coronavirus-infected pneumonia in Wuhan, China. JAMA 2020; 323 (11): 1061-1069. https:// www.ncbi.nlm.nih.gov/pmc/articles/PMC7042881/

69. Smart Patients. Various combination of Protease Inhibitors, Oseltamivir, Favipiravir, and Chloroquin for Treatment of COVID-19: A Randomized Control Trial. https://www. smartpatients.com/trials/NCT04303299

70. Hennon T, et, al. COVID-19 associated Multisystem Inflammatory Syndrome in Children (MIS-C) guidelines; a Western New York approach. Progress in Pediatric Cardiology 2020; 57: 101232. https://doi.org/10.1016/j. ppedcard.2020.101232

71. Fu B, et al. Why tocilizumab could be an effective treatment for severe COVID-19? J TransI Med 2020; 18 (1): 164. doi. 10.1186/s12967-020-02339-3

72. Zhang $C$, et al. Cytokine release syndrome in severe COVID-19: interleukin-6 receptor antagonist tocilizumab may be the key to reduce mortality. Int J Antimicrob Agents 2020; 55 (5): 105954. doi. 10.1016/j.ijantimicag.2020.105954.

73. Luo $\mathrm{P}$, et al. Tocilizumab treatment in COVID-19: A single center experience. J Med Virol 2020; 92 (7): 814-818. doi. 10.1002/jmv.25801. 
Rodríguez-Herrera R, et al. SARS-CoV-2 (COVID-19) en pacientes pediátricos

74. Kashiouris $\mathrm{M}$, et al. The emerging role of vitamin $\mathrm{C}$ as a treatment for sepsis. Nutrients 2020; 12 (2): 1-16. doi. 10.3390/nu12020292

75. National Library of Medicine. Fingolimod in COVID-19. https://clinicaltrials.gov/ct2/show/NCT04280588?term= NCT04280588\&draw $=2 \&$ rank $=1$

76. Wu R, et al. An Update on current therapeutic drugs treating COVID-19. Curr Pharmacol Rep 2020: 1-15. doi. 10.1007/s40495-020-00216-7.
77. Foods and Drugs Administration. Recommendations for Investigational COVID-19 Convalescent Plasma. https:// www.fda.gov/vaccines-blood-biologics/investigational-newdrug-ind-or-device-exemption-ide-process-cber/recommendations-investigational-covid-19-convalescent-plasma

78. Folegatti $P$, et al. Safety and immunogenicity of the ChAdOx1 $\mathrm{nCoV}-19$ vaccine against SARS-CoV-2: a preliminary report of a phase $1 / 2$, single-blind, randomized controlled trial. Lancet 2020; 396 (10249): 467-478. doi. 10.1016/ S0140-6736(20)31604-4 